\title{
Permutation of permutations and Cayley's theorem
}

\author{
Open Mathematics Collaboration*广
}

April 16, 2021

\begin{abstract}
The idea behind this preliminary white paper is to try to understand the multiplication of the natural numbers as permutations. For this end, we construct a set of the permutation of permutations of the symmetric group $S_{3}$.
\end{abstract}

keywords: symmetric group, permutations, abstract algebra

The most updated version of this white paper is available at https://osf.io/hd6ar/download

\section{Introduction}

1. This white paper is waiting peer review and should therefore be treated as preliminary.

2. It is part of the global scholarly ecosystem published in the OJMP.

3. Online version https://bit.1y/3tjxcF7

*All authors with their affiliations appear at the end of this white paper.

†Corresponding author: mplobo@uft.edu.br|Open Mathematics Collaboration 


\section{Open Invitation}

Review, add content, and co-author this white paper [6,7].

Join the Open Mathematics Collaboration.

Send your contribution to mplobo@uft.edu.br.

\section{Open Science}

The latex file for this white paper together with other supplementary files are available in [8].

\section{Ethical conduct of research}

This original work was pre-registered under the OSF Preprints [9], please cite it accordingly [10]. This will ensure that researches are conducted with integrity and intellectual honesty at all times and by all means.

\section{Acknowledgements}

+ Center for Open Science https://cos.io

+ Open Science Framework https://osf.io

\section{Agreement}

4. All authors agree with [7]. 


\section{References}

[1] Velleman, Daniel J. How to prove it: A structured approach. Cambridge University Press, 2019.

https://books.google.com/books?vid=ISBN0521861241

[2] Warner, Steve. Abstract Algebra for Beginners. GET 800, 2018.

https://books.google.com/books?id=UFleyAEACAAJ

[3] Dixon, John D., and Brian Mortimer. Permutation groups. Vol. 163. Springer Science \& Business Media, 1996.

https://books.google.com/books?vid=1SPjBwAAQBAJ

[4] Dummit, David Steven, and Richard M. Foote. Abstract Algebra. Vol. 3. Hoboken: Wiley, 2004.

https://books.google.com/books?id=znzJygAACAAJ

[5] Rotman, Joseph J. A first course in abstract algebra. Pearson College Division, 2000.

https://books.google.com/books?id=ctEZAQAAIAAJ

[6] Lobo, Matheus P. "Microarticles." OSF Preprints, 28 Oct. 2019. https://doi.org/10.31219/osf .io/ejrct

[7] Lobo, Matheus P. "Simple Guidelines for Authors: Open Journal of Mathematics and Physics." OSF Preprints, 15 Nov. 2019.

https://doi.org/10.31219/osf .io/fk836

[8] Lobo, Matheus P. "Open Journal of Mathematics and Physics (OJMP)." OSF, 21 Apr. 2020.

https://doi.org/10.17605/osf .io/6hzyp

https://osf.io/6hzyp/files

[9] COS. Open Science Framework. https://osf .io 
[10] Lobo, Matheus P. "Permutation of Permutations and Cayley's Theorem." OSF Preprints, 14 Apr. 2021.

https://doi.org/10.31219/osf .io/hd6ar

[11] Lobo, Matheus P. "Composition of Permutations in the Symmetric Group." OSF Preprints, 16 Apr. 2021.

https://doi.org/10.31219/osf .io/r3jvu

\section{The Open Mathematics Collaboration}

Matheus Pereira Lobo (lead author, mplobo@uft.edu.br) $)^{1,2}$ https://orcid.org/0000-0003-4554-1372

${ }^{1}$ Federal University of Tocantins (Brazil)

${ }^{2}$ Universidade Aberta (UAb, Portugal) 


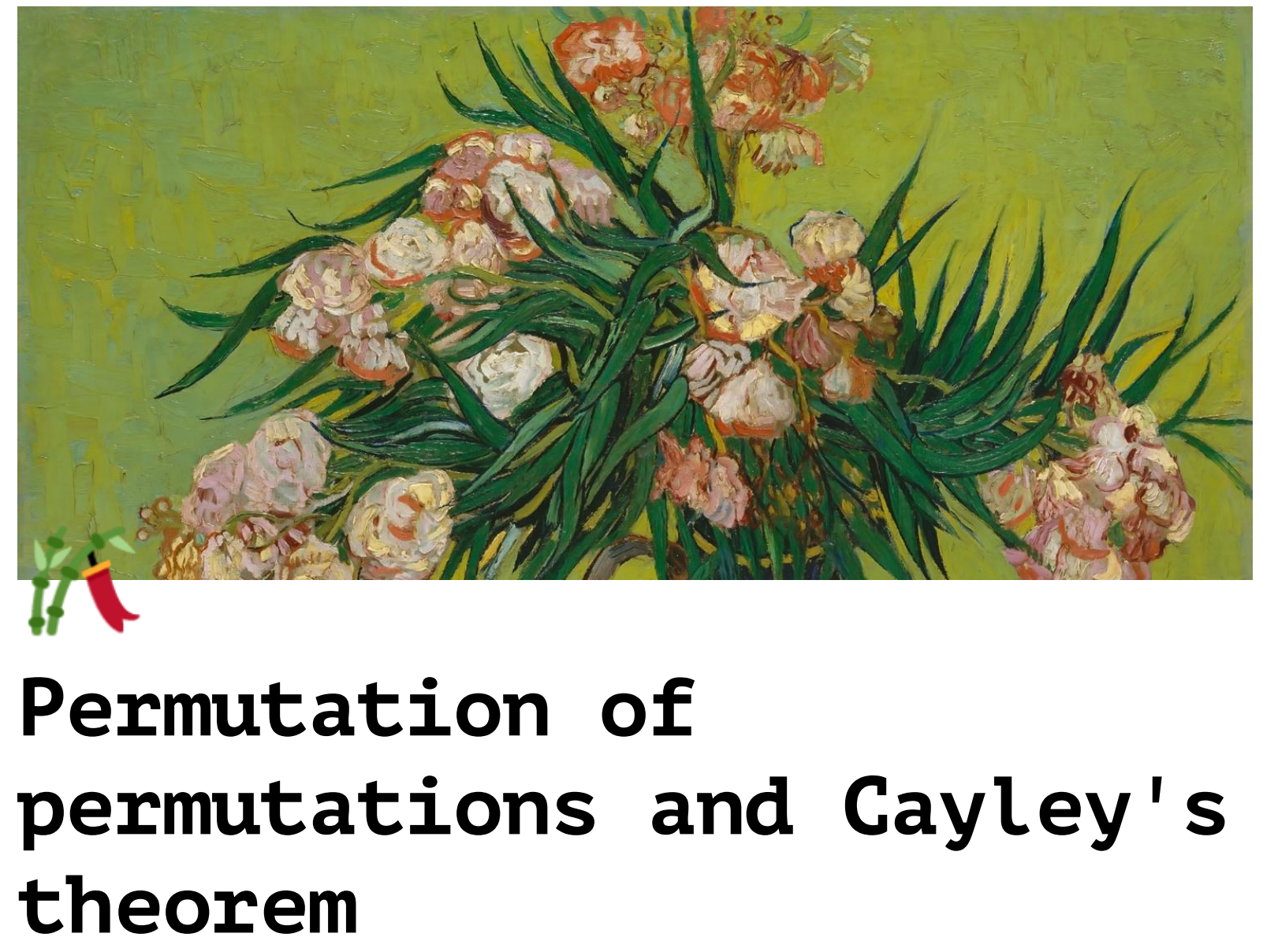

https://doi.org/10.31219/osf.io/hd6ar

Published 14 Apr 2021

Volume 3, Article 226 (IW)

DOWNLOAD PDF

s 1 . Abstract

2. Prerequisites

-3 . Group

4. Cayley's theorem

2 5. Permutation of permutations

6. Final Remarks

Q 7. References 


\section{Abstract}

The idea behind this preliminary white paper is to try to understand the multiplication of the natural numbers as permutations. For this end, we construct a set of the permutation of permutations of the symmetric group $S_{3}$. 


\section{Prerequisites}

it Function

One-to-one function (injection).

$\div 0$ Onto function (surjection).

to Ordered pair

Cartesian product

2 Binary operation

Bijective function

(- Permutation

Homomorphism

(5) Isomorphism 


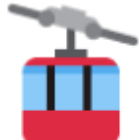

\section{Function}

Function from $A$ to $B$

$$
\begin{gathered}
f: A \rightarrow B \\
\forall a \in A \exists ! b \in B((a, b) \in f)
\end{gathered}
$$

$f, A, B:=$ sets

$\exists$ ! := exists exactly one

$(a, b):=$ ordered pair

[1] 
采言

One-to-one function (injection)

$f: A \rightarrow B$

$$
\neg \exists a_{1} \in A \exists a_{2} \in A\left(f\left(a_{1}\right)=f\left(a_{2}\right) \wedge a_{1} \neq a_{2}\right)
$$

[1] 


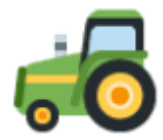

Onto function

(surjection)

$f: A \rightarrow B$

$$
\forall b \in B \exists a \in A(f(a)=b)
$$

[1] 
tos

Ordered pair

$$
(a, b)=\{\{a\},\{a, b\}\}
$$

$a:=$ first coordinate

$b:=$ second coordinate

$[1,2]$ 


\section{Cartesian product}

$A \times B=\{(a, b) \mid a \in A, b \in B\}$

$A, B:=$ sets

$(a, b):=$ ordered pair

[1] 


\section{Binary operation}

$$
\star: S \times S \rightarrow S
$$

$$
\begin{aligned}
& S:=\text { set } \\
& S \times S:=\text { Cartesian product }
\end{aligned}
$$

[2] 


\section{Bijective function}

Bijective function := one-to-one + onto

[1] 


\section{Permutation}

Permutation of $A:=$ bijection from $A$ to itself [3] 


\section{Homomorphism}

$$
f^{h}
$$

$f^{h}: G \rightarrow H$

$\forall x, y \in G: f^{h}(x * y)=f^{h}(x) \circ f^{h}(y)$

$f^{h}:=$ function

$G, H:=$ sets

$\star, \circ:=$ binary operations

$(G),,(H, \circ):=$ groups

$[2,4,5]$ 


\section{(2) \\ Isomorphism}

Isomorphism := bijective homomorphism

$[2,4,5]$ 


\section{Group}

$$
(G, \star)
$$

Associativity: $\forall x, y, z \in G,(x \star y) \star z=x \star(y \star z)$

Identity: $\exists e \in G: \forall x \in G, e \star x=x \star e=x$

Inverse: $\forall x \in G \exists y \in G: x \star y=y \star x=e$

$G:=$ set

$\star:=$ binary operation

[2] 


\section{Cayley's theorem}

$$
(G, \star) \cong\left(P, \circ_{\alpha}\right)
$$

$(G, \star):=$ group

$\left(P, \circ_{\alpha}\right):=$ permutation group

$\cong$ isomorphism

$\star, \circ_{\alpha}:=$ binary operations

$\alpha: P \rightarrow P$ (permutation $:=$ bijective function)

$\circ_{\alpha}:=$ composition of permutations

$[2,3]$ 


\section{2 \\ 5. Permutation of permutations}

Let $N_{3}=\{1,2,3\}$. Suppose $\left(N_{3}, \star\right)$ is a group.

$S_{3}:=$ group of all the permutations of $N_{3}$

$S_{3}=\{(1),(12),(13),(23),(123),(132)\}$

From Cayley's theorem, there is a bijection between $\left(N_{3}, \star\right)$ and a permutation group.

Brainstorming: constructing the set PP3

輩 Multiplication tables for S2 and S3 


\section{Brainstorming:}

constructing the set PP3

$S_{3}=\{(1),(12),(13),(23),(123),(132)\}$

Let $P P_{3}=\{((1,1)),((2,2)),((3,3)),((12,2)),((13,3)),((23,6))$, $((123,6)),((132,6))\}$.

$((1,1))$ is the permutation of $(1)$ by $(1)$.

$((2,2))$ is the permutation of $(2)$ by $(2)$.

$((3,3))$ is the permutation of $(3)$ by $(3)$.

$((12,2))$ is the permutation of $(12)$ by $(2)$.

$((13,3))$ is the permutation of $(13)$ by $(3)$.

$((23,6))$ is the permutation of $(23)$ by $(6)$.

$((123,6))$ is the permutation of $(123)$ by $(6)$.

$((132,6))$ is the permutation of $(132)$ by $(6)$.

Clearly there is a bijection between $S_{3}$ and $P P_{3}$ 
Multiplication tables for S2 and S3

Check [11] at https://doi.org/10.31219/osf.io/r3jvu or here.

$S_{2}=\{(1),(12)\}$, let $a:=(1)$ and $b:=(12)$

\begin{tabular}{c|cc}
$S_{2}$ & $a$ & $b$ \\
\hline$a$ & $a$ & $b$ \\
$b$ & $b$ & $a$
\end{tabular}

$S_{3}=\{(1),(12),(13),(23),(123),(132)\}$
$a:=(1), \quad b:=(12), \quad c:=(13), \quad d:=(23), \quad e:=(123)$
\begin{tabular}{c|cccccc}
$S_{3}$ & $a$ & $b$ & $c$ & $d$ & $e$ & $f$ \\
\hline$a$ & $a$ & $b$ & $c$ & $d$ & $e$ & $f$ \\
$b$ & $b$ & $a$ & $e$ & $f$ & $c$ & $d$ \\
$c$ & $c$ & $f$ & $a$ & $e$ & $d$ & $b$ \\
$d$ & $d$ & $e$ & $f$ & $a$ & $b$ & $c$ \\
$e$ & $e$ & $d$ & $b$ & $c$ & $f$ & $a$ \\
$f$ & $f$ & $c$ & $d$ & $b$ & $a$ & $e$
\end{tabular}




\section{Final Remarks}

We present a bijection from $P P_{3}$ (the permutation of permutations of $N_{3}$ ) and a group $\left(\mathbb{N}_{3}, \star\right)$ in order to have an insight of $\left(\mathbb{N}_{3}, \star\right)$ as a permutation group, a known result from Cayley's theorem. 


\section{References}

1. Velleman, Daniel J. How to prove it: A structured approach. Cambridge University Press, 2019.

https://books .google.com/books?vid=ISBN0521861241

2. Warner, Steve. Abstract Algebra for Beginners. GET 800, 2018. https://books.google.com/books?id=UFleyAEACAAJ\}

3. Dixon, John D., and Brian Mortimer. Permutation groups. Vol. 163. Springer Science I\& Business Media, 1996. https: //books.google.com/books?vid=1SPj BWAAQBAJ

4. Dummit, David Steven, and Richard M. Foote. Abstract Algebra. Vol. 3. Hoboken: Wiley, 2004. https://books.google.com/books?id=znzJy.gAACAAJ

5. Rotman, Joseph J. A first course in abstract algebra. Pearson College Division, 2000.

https: //books.google.com/books?id=ctEZAQAAIAAJ

6. Lobo, Matheus P. "Microarticles." OSF Preprints, 28 0ct. 2019. https://doi.org/10.31219/osf.io/ejrct

7. Lobo, Matheus P. "Simple Guidelines for Authors: Open Journal of Mathematics and Physics." OSF Preprints, 15 Nov. 2019. https://doi.org/10.31219/osf.io/fk836

8. Lobo, Matheus P. "Open Journal of Mathematics and Physics (OJMP)." OSF, 21 Apr. 2020.

https://doi.org/10.17605/osf.io/6hzyp\}

https://osf.io/6hzyp/files

9. CoS. Open Science Framework. https://osf.io

.0. Lobo, Matheus P. "Permutation of Permutations and Cayley's Theorem." OSF Preprints, 14 Apr. 2021. https://doi.org/10.31219/osf.io/hd6ar 
1. Lobo, Matheus P. "Composition of Permutations in the Symmetric Group." OSF Preprints, 16 Apr. 2021. https://doi.org/10.31219/osf.io/r3jvu 